\title{
Secondary Metabolites Produced by an Endophytic Fungus Pestalotiopsis microspora
}

\section{G. R. Nalin Rathnayake ${ }^{1}$ N. Savitri Kumar ${ }^{1} \cdot$ Lalith Jayasinghe $^{1} \cdot$ Hiroshi Araya $^{2} \cdot$ Yoshinori Fujimoto $^{1,2}$}

Received: 1 July 2019 / Accepted: 3 November 2019 / Published online: 15 November 2019

(C) The Author(s) 2019

\section{Abstract}

An endophytic fungus Pestalotiopsis microspora isolated from the fruits of Manilkara zapota was cultured in potato dextrose broth media. Chromatographic separation of the EtOAc extract of the broth and mycelium led to the isolation of a new azaphilonoid named pitholide E (1), in addition to previously identified pitholide B (2), pitholide D (3), pestalotin (LL-P880 $\alpha$ ) (4), PC-2 (5), LL-P880ß (6), tyrosol (7) and 4-oxo-4H-pyran-3-acetic acid (8). An endophytic fungus $P$. microspora from $M$. $z a p o t a$ and the isolation of compounds 1-5, $\mathbf{7}$ and $\mathbf{8}$ from $P$. microspora are reported here for the first time.

\section{Graphic Abstract}

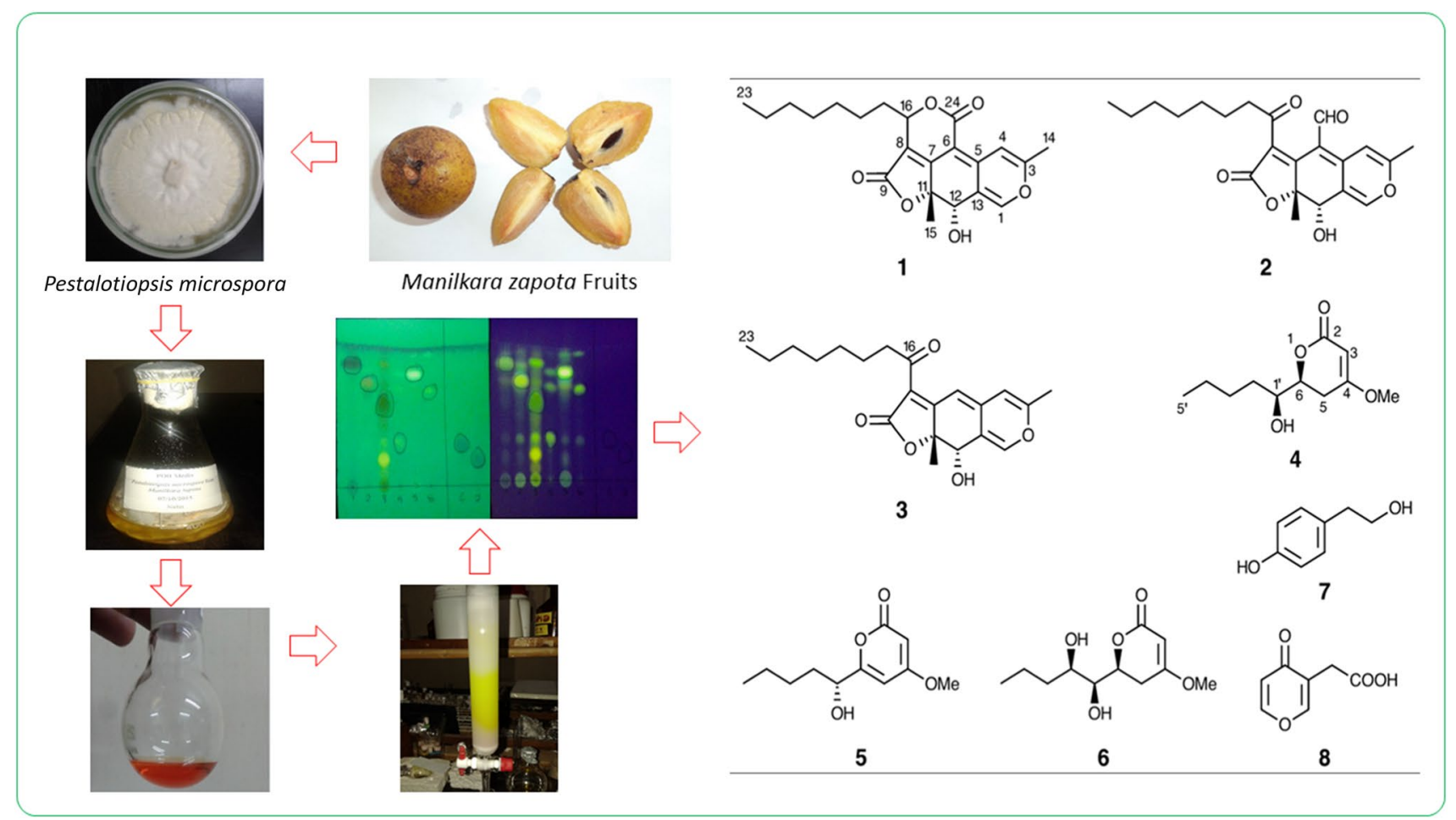

Keywords Pestalotiopsis microspora $\cdot$ Manilkara zapota $\cdot$ Endophyte $\cdot$ Pitholide E $\cdot$ Pestalotin

Lalith Jayasinghe

ulbj2003@yahoo.com; lalith.ja@nifs.ac.lk

Extended author information available on the last page of the article 


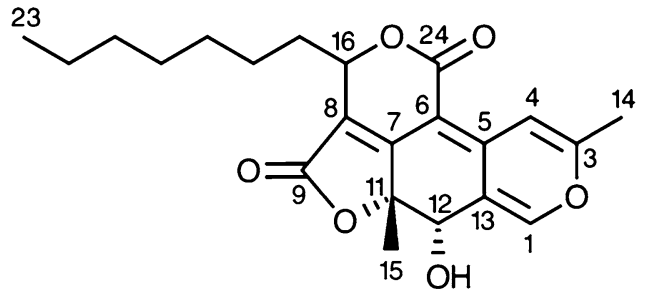

1<smiles>CCCCCCCC(=O)C1=C2C=C3C=C(C)OC=C3[C@@H](O)[C@@]2(C)OC1=O</smiles><smiles>CCCCCCCC(=O)C1=C2C(C=O)=C3C=C(C)OC=C3[C@@H](O)[C@@]2(C)OC1=O</smiles>

2<smiles>CCCC[C@@H](O)[C@H]1CC(OC)=CC(=O)O1</smiles>

4
3<smiles>CCCC[C@H](O)c1cc(OC)cc(=O)o1</smiles>

5<smiles>CCC[C@H](O)[C@H](O)[C@H]1CC(OC)=CC(=O)O1</smiles>

6<smiles>OCCc1ccc(O)cc1</smiles>

7<smiles>O=C(O)Cc1coccc1=O</smiles>

8

Fig. 1 Structures of compounds $\mathbf{1}-\mathbf{8}$

\section{Introduction}

Fungi are considered to be an important source of bioactive compounds. Fungi can be categorized into two groups, epiphytic fungi and endophytic fungi. Epiphytic fungi live on the surface of a host while endophytic fungi colonize inner tissues and even live in the cells of their hosts [1]. Some endophytic fungi have developed the ability to produce the same or similar bioactive substances as those originating from the host plants [2]. We have previously reported several bioactive compounds produced by endophytes isolated from Sri Lankan plants [3-10]. Recently we investigated the secondary metabolites produced by an endophytic fungus Pestalotiopsis microspora, isolated from the fruits of Manilkara zapota (local name: Sapodilla) of the family Sapotaceae. The fruits are popular in tropical countries and the leaves are used for antidiabetic treatment. Various antidiabetic and antilipidemic effects of M. zapota fruits have been reported [11]. In addition, the traditional uses, phytochemistry and pharmacological activity of the plant have been reviewed [12]. In this paper we report the isolation and identification of a new azaphilonoid named pitholide E (1) from a culture of $P$. microspora, in addition to already known pitholide $\mathrm{B}$ (2), pitholide D (3), pestalotin (LL-P880 $\alpha$ ) (4), PC-2 (5), LL-P880 $\beta$ (6), tyrosol (7) and 4-oxo-4H-pyran-3-acetic acid (8) (Fig. 1).

\section{Results and Discussion}

An endophytic fungus isolated from the fruits of $M$. zapota was identified as $P$. microspora by morphological studies (cotton like mycelium grown with irregular margins, uniform conidia consists with five cells with three brown to fuliginous median cells and hyaline end cells) as well as by sequence analysis of the ITS region of the rDNA gene. Amplification of the ITS region was carried out using the universal eukaryotic primers of ITS1 and ITS4. BLAST search indicated that the sequence of the ITS region had $100 \%$ similarity to that of $P$. microspora JD1 (GenBank 
Accession No. KP231875.1). A pure culture of P. microspora (IFS/N/MZ/1/2014) and photographic evidence of the fruits of $M$. zapota and the strain are deposited at the National Institute of Fundamental Studies.

Silica gel TLC autobiography analysis [13] found that EtOAc extract obtained from the culture broth and from mycelium of $P$. microspora grown in potato dextrose broth (PDB) displayed antifungal activity against $C$. cladosporioides. In addition, antioxidant activity was also demonstrated by DPPH radical scavenging assay $\left(\mathrm{IC}_{50} 63.5 \mu \mathrm{g} /\right.$ $\mathrm{mL}$ ) [14]. However, it was inactive in the assays of brine shrimp lethality [15], phytotoxicity against Lactuca sativa (inhibition of root and shoot growth) [16], $\alpha$-amylase inhibition [17] and anticandidal activity against Candida tropicalis [18]. Chromatographic separation of the EtOAc extract over silica gel and Sephadex LH-20, and by preparative silica gel TLC furnished a new azaphilonoid pitholide E (1), in addition to previously identified pitholide $\mathrm{B}(\mathbf{2})$, pitholide $\mathrm{D}(\mathbf{3})$ [19], pestalotin (4) [20, 21], PC-2 (5) [22, 23], LL-P880ß (6) $[24,25]$, tyrosol (7) [26] and 4-oxo-4H-pyran-3-acetic acid (8) $[27,28]$. The structures of the known compounds were determined by comparing their spectroscopic data with literature values. TLC analysis indicated that none of these compounds were present in the $\mathrm{MeOH}$ extract of M. zapota fruits.

Compound 1 was isolated as luminous yellow oil. HRFABMS data indicated that it has a molecular formula of $\mathrm{C}_{22} \mathrm{H}_{26} \mathrm{O}_{6}$, with 10 degrees of unsaturation. The ${ }^{1} \mathrm{H}$ and ${ }^{13} \mathrm{C}$ NMR spectra, showing 22 carbon signals, resembled those of an azaphilonoid such as pitholide B (2) [19]. Compound 1 displayed three methyl signals at $\delta 2.29$ (s), 1.44 (s) and $0.87\left(\mathrm{t}, J=7.0 \mathrm{~Hz}\right.$ ) assignable to $\mathrm{H}_{3}-14, \mathrm{H}_{3}-15$ and $\mathrm{H}_{3}-23$, respectively, in the ${ }^{1} \mathrm{H}$ NMR spectrum. In addition, the presence of an oxymethine proton at $\delta 4.53(\mathrm{~s}, \mathrm{H}-12)$ and two significantly deshielded olefinic protons at $\delta 7.54$ (s, $\mathrm{H}-1)$ and 7.61 (s, H-4), supported $\mathrm{a}_{22}$-pitholide structure. Detailed analysis of the NMR spectra in comparison with those of compound 2 suggested that the $\mathrm{C}-24$ aldehyde in 2 was oxidized into an acid (appearance of $\delta_{\mathrm{C}} 162.6$ in 1 with disappearance of $\delta_{\mathrm{H}} 9.71 / \delta_{\mathrm{C}} 186.8$ in 2), while the C-16 ketone in 2 was reduced to an alcohol (appearance of $\delta_{\mathrm{H}} 5.46$ $(\mathrm{dd}, J=7.0,3.5 \mathrm{~Hz}) / \delta_{\mathrm{C}} 79.1$ in 1 with disappearance of $\delta_{\mathrm{C}}$ 198.6 in 2 ), and these were cyclized to form a $\delta$-lactone. The lactonization was supported by an HMBC correlation from H-16 to C-24 and a downfield shifted H-16 $(\delta 5.46)$ compared to that of an allylic secondary alcohol. Key HMBC correlations for compound 1 (Fig. 2) were consistent with the proposed tetra-cyclic structure. Complete ${ }^{1} \mathrm{H}$ and ${ }^{13} \mathrm{C}$ NMR assignments of compound $\mathbf{1}$, attained with assistance of the HMQC spectrum, are described below.

Compound $\mathbf{1}$ is stereochemically homogenous at the $\mathrm{C}-16$ position as evidenced by a single set of ${ }^{13} \mathrm{C}$ NMR resonances. NOE correlation between $\mathrm{H}_{3}-15$ and $\mathrm{H}-16$ was

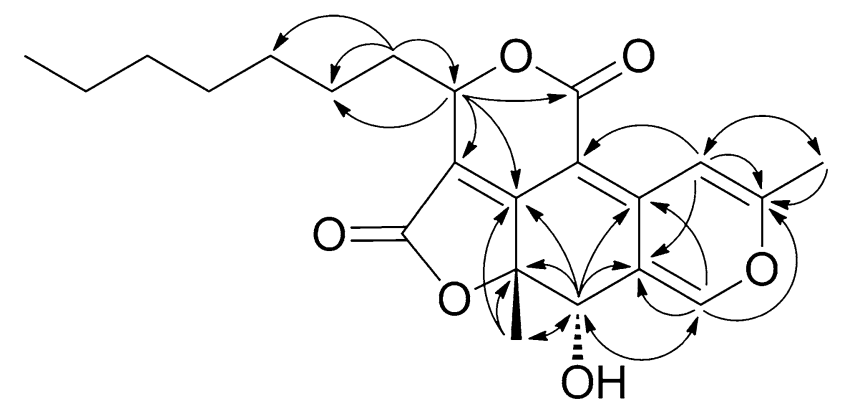

Fig. 2 Key HMBC correlations for compound 1

not observed in the NOE experiments (ROESY and differential NOE spectra irradiating $\mathrm{H}_{3}-15$ and $\mathrm{H}-16$ ), although an intense NOE correlation was observed between $\mathrm{H}_{3}-15$ and $\mathrm{H}-12$. These findings may suggest an anti relationship between $\mathrm{H}_{3}-15$ and $\mathrm{H}-16$ (the proximity of $\mathrm{H}-16$ and the nearest proton of $\mathrm{H}_{3}-15$ was calculated as $4.3 \AA$ for the $s y n$ isomer). Regarding the absolute stereochemistries at the $\mathrm{C}-11$ and $\mathrm{C}-12$ positions of pitholide $\mathrm{E}, 11 R, 12 S$ configuration was proposed for monaphilols $\mathrm{A}$ and $\mathrm{B}$, which have the same partial structure (trans-orientation of 11-Me and 12-hydroxy groups) as pitholides $\mathrm{B}, \mathrm{D}$ and $\mathrm{E}$, in view of a large negative sign of specific rotation [29]. The assignment is based on the assumption that the absolute configuration at the $\mathrm{C}-11$ chiral center contributes the negative specific rotation. The assumption will be acceptable since structurally related $11 R$ azaphilonoids (cis-orientation of 11-methyl and 12-hydroxy groups) such as rotiorinol $\mathrm{A}$ as well as $11 R$ azaphilonoids with a 12-oxo group such as monascorubrin, rubropunctatin and (-)-rotiorin display a large negative specific rotation commonly (Fig. 3). The $11 R$ configuration of rotiorinol $\mathrm{A}$ and (-)-rotiorin have been established unambiguously [30]. Hence, we propose $11 R, 12 S$ configuration for pitholide $\mathrm{B}, \mathrm{D}$ as well as pitholide $\mathrm{E}$, although the stereochemical assignments should be ascertained by an unambiguous manner such as X-ray and ECD study. Our attempt to determine the absolute configuration of pitholide $\mathrm{D}$ using Mosher ester method was not successful since irregularities in the $\Delta(\delta S-\delta R)$ values were observed. Finally, our specimens of pitholides $\mathrm{B}$ and $\mathrm{D}$ showed much larger negative specific rotations $\left([\alpha]_{D}{ }^{25}-741(c, 0.23, \mathrm{MeOH})\right.$ for 2 and $-980(c, 0.077, \mathrm{MeOH})$ for 3 than the reported values $\left([\alpha]_{\mathrm{D}}-158(c, 0.85, \mathrm{MeOH})\right.$ for 2 and $[\alpha]_{\mathrm{D}}-248(c, 0.51$, $\mathrm{MeOH}$ ) for 3 [19]).

Pestalotiopsis microspora is known as a taxol-producing fungus [31] and reported to have an ability to break down and digest polyurethane [32]. Pitholides B (2) and D (3) have been previously isolated, along with pitholides A and C, from Pithomyces sp. derived from a marine tunicate [19], and they structurally belong to an azaphilone class of polyketides. These pitholides are assumed to be biologically 
Fig. 3 Correlation between C-11 configuration and a large negative specific rotation

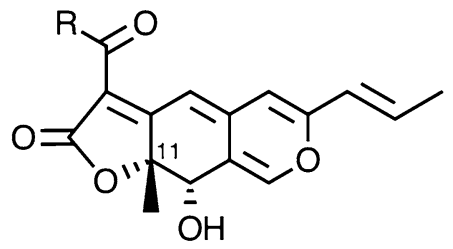

$\mathrm{R}=n-\mathrm{C}_{7} \mathrm{H}_{15}$ monaphilol $\mathrm{A} \quad[\mathrm{a}]_{\mathrm{D}}-2649$ $\mathrm{R}=n-\mathrm{C}_{5} \mathrm{H}_{11}$ monaphilol $\mathrm{B}$<smiles>[R]C(=O)C1=C2C=C3C=C(/C=C/C)OC=C3C(=O)[C@@]2(C)OC1=O</smiles>

$\mathrm{R}=n-\mathrm{C}_{7} \mathrm{H}_{15}$ monascorubrin $[\mathrm{a}]_{\mathrm{D}}-3390$ $\mathrm{R}=n-\mathrm{C}_{5} \mathrm{H}_{11}$ rubropunctatin $[\mathrm{a}]_{\mathrm{D}}-3670$<smiles>CC[C@H](C)/C=C(C)/C=C/C1=CC2=CC3=C(C(C)=O)CO[C@]3(C)C(=O)C2=CO1</smiles>

(-)-rotiorin $[a]_{D}-2251$

$\uparrow$ chemical conversion<smiles>CC[C@H](C)/C=C(C)/C=C/C1=CC2=CC3=C(C(C)=O)C(=O)O[C@@]3(C)[C@H](O)C2=CO1</smiles>

rotiorinol $A[a]_{D}-2261$

(established by Mosher ester method) benign, for example, inactive in brine shrimp lethality assay [19]. (-)-Pestalotin (6S,1'S-form) (4) was first isolated from Pestalotia cryptomeriaecola [33] and has since been identified in several fungi including Pestalotiopsis sp. such as $P$. karstenii [34]. Epipestalotin (either $6 R, 1^{\prime} S$ - or $6 S, 1^{\prime} R$-form) was reported from an endophytic fungus isolated from a tropical lichen Everniastrum nepalense [35]. (+)-(1'R)-PC-2 (5) which is also called dehydropestalotin has been reported from some endophytic fungi including $P$. mangiferae [36] and Penicillium sp.[22]. Specific rotation of $5\left([\alpha]_{\mathrm{D}}{ }^{25}+76.5\right.$ $(c, 0.62, \mathrm{MeOH}))$ was found to be identical to the literature value $\left([\alpha]_{\mathrm{D}}+78.5(c, 0.19, \mathrm{MeOH})\right)[22]$, implying it had a $1^{\prime} R$ configuration. It is noteworthy that the $\mathrm{C}-1$ ' configuration of $\mathbf{5}$ is opposite to those of compounds $\mathbf{4}$ and $\mathbf{6}$, even though compounds 4-6 are produced by the same fungus strain. The antipode of 5 (1'S-form) $\left([\alpha]_{\mathrm{D}}-77.1(c, 0.52, \mathrm{MeOH})\right.$ has also been identified in a few other fungi including $P$. phitiniae [37] and Urnula craterium [38]. (-)-LL-P880 $\beta$ (6S,1'S,2' $R$-form) (6) has been previously reported from some fungi including $P$. microspora isolated from Taxus chinensis [39]. The C-2' epimer of 6 ( $6 S, 1^{\prime} S, 2^{\prime} S$-form) has been also reported from an endophytic fungus $P$. virgatula obtained from mangroves [40]. Compound 4 was reported to act as a plant growth regulator, as a gibberellin synergist [33], while compounds $\mathbf{4}$ and $\mathbf{6}$ have moderate cytotoxicity towards several cancer cell lines [34, 41]. Tyrosol (7) is a commonly occurring fungal metabolite and has been isolated from endophytic fungi Pestalotiopsis sp. including P. versicolor [42] and P. virgatula [43]. The tyrosine metabolite is widespread in Candida species and reported as a quorumsensing molecule in the yeast $C$. albicans [44]. 4-Oxo-4Hpyran-3-acetic acid (8) has been isolated from several fungi including Pestalotiopsis sp [45]. Compounds 1, 4, 6 and 7 were evaluated for antioxidant and antifungal activity against C. cladosporioides, but none of them showed any significant activity. In conclusion, the endophytic fungus $P$. microspora was isolated from $M$. zapota for the first time, whereas $P$. gracilis was isolated previously from the leaf spots of Achras sapota (syn. M. sapota) [46]. Chemical investigation of the secondary metabolites produced by the fungus led to the isolation of a new azaphilonoid pitholide $\mathrm{E}(\mathbf{1})$, which possesses 22 carbon atoms and an extra $\delta$-lactone ring. In addition, two rare azaphilonoids, pitholide B (2) and pitholide D (3), were isolated from the genus Pestalotiopsis for the first time. Also, this is the first isolation of compounds 4, 5, 7 and $\mathbf{8}$ from $P$. microspora, although they have been previously isolated from Pestalotiopsis species. The current study only investigated the fruits of M. zapota, so it would be interesting to study endophytic fungal diversity in other parts of $M$. zapota since the secondary metabolites produced by endophytic fungi from this medicinal plant are not well studied.

\section{Experimental}

\subsection{General}

Extractions were performed using a sonicator (VWR Ultrasound cleaner, model-USC 1700D). Analytical TLC was carried out with silica gel $60 \mathrm{~F}_{254}$ precoated aluminum sheets (Merck Art. 1.05554). Compounds on TLC were located using a UV lamp and by heating after spraying with acidic anisaldehyde. Silica gel (Fluka 60741, Merck Art. 7734 and 9385) and Sephadex LH-20 were used for column chromatography. Preparative thin layer chromatography (PTLC) was carried out using silica gel $60 \mathrm{~F}_{254}$ precoated glass plates 
(Merck Art 1.05715). ${ }^{1} \mathrm{H}$ NMR and ${ }^{13} \mathrm{C}$ NMR were recorded on a Bruker DRX500 $\left(500 \mathrm{MHz}\right.$ for ${ }^{1} \mathrm{H}$ and $125 \mathrm{MHz}$ for $\left.{ }^{13} \mathrm{C}\right)$ or JEOL JMN-AL300 $\left(300 \mathrm{MHz}\right.$ for ${ }^{1} \mathrm{H}$ and $75 \mathrm{MHz}$ for ${ }^{13} \mathrm{C}$ ) spectrometer in $\mathrm{CD}_{3} \mathrm{OD}$ or $\mathrm{CDCl}_{3}$ solution at $20^{\circ} \mathrm{C}$. Optical rotations were measured on a JASCO P-2200 polarimeter. IR spectra were measured on a JASCO FT/IR-460 spectrometer. UV spectra were recorded on a JASCO MD-4017 photo diode array detector. Positive-ion FABMS were obtained on a JEOL JMX-AX505HA spectrometer.

\subsection{Isolation of Endophytic Fungus}

Healthy and mature M. zapota fruits were purchased from the local market in Kandy, Sri Lanka in March 2014. Fruits were rinsed with running water and triple sterilized with ethanol, $5 \% \mathrm{NaOCl}$ and distilled water. Interior segments (ca. $10 \mathrm{~mm} \times 5 \mathrm{~mm}$ ) of sterilized fruits were placed on PDA media in Petri dishes and incubated at room temperature for 5 days. Emerging fungi were serially transferred to PDA media on Petri dishes to obtain pure culture of endophytic fungus.

\subsection{Fermentation of Fungus, Extraction, Bioassays and Isolation of Compounds}

An endophytic fungus $P$. microspora isolated from the $M$. zapota was cultured by inoculating pure culture grown on potato dextrose agar, in eighty $1 \mathrm{~L}$-conical flasks each containing $400 \mathrm{~mL}$ of PDB medium. The flasks were allowed to stand at room temperature for initial 10 days, and shaked every other day on a laboratory shaker $(100 \mathrm{rpm})$. The fermentation medium was filtered after one month and the filtrate was extracted with EtOAc. The residual mycelium was extracted with EtOAc using sonicator. The two EtOAc extracts were combined $(23.5 \mathrm{~g})$ since they showed almost identical TLC pattern. The combined EtOAc extract was screened for antioxidant activity using DPPH assay [14], $\alpha$-amylase inhibitory activity [17], antifungal activity against Cladosporium cladosporioides by TLC bioautography method [13], anticandidal activity against five species (C. albicans, C. parapsilosis, C. glabrata, C. krusei and $C$. tropicalis) [18], phytotoxicity against $L$. sativa seed germination [16] and brine shrimp toxicity against Artemia salina [15]. Chromatographic separation of the combined EtOAc extract was carried out over silica gel (n-hexane-EtOAc-MeOH/n-hexane- $\mathrm{CH}_{2} \mathrm{Cl}_{2}-\mathrm{MeOH}$ ) and Sephadex LH-20 (MeOH), and by PTLC to furnish eight compounds, pitholide $\mathrm{E}$ (1) (19 mg), together with known pitholide B (2) (58 mg), pitholide D (3) (26 mg), pestalotin (LL-P880 $\alpha$ ) (4) (6 mg), PC-2 (5) (18 mg), LL-P880ß (6) (28 mg), tyrosol (7) (46 mg) and 4-oxo-4H-pyran-3-acetic acid (8) (24 mg).

Pitholide E (1): luminous yellow oil; $[\alpha]_{\mathrm{D}}^{25}-1080(c$, 0.055, MeOH); UV $\lambda_{\max }(\mathrm{MeOH}): 205,254,294,401 \mathrm{~nm}$;
IR $\nu_{\text {max }}\left(\mathrm{CHCl}_{3}\right) 3400,2958,2928,2856,1750,1682,1649$, $1550 \mathrm{~cm}^{-1} ;{ }^{1} \mathrm{H}$ NMR $\left(\mathrm{CDCl}_{3}, 500 \mathrm{MHz}\right): \delta 7.61(\mathrm{~s}, \mathrm{H}-4)$, 7.54 (s, H-1), 5.46 (dd, $J=7.0,3.5 \mathrm{~Hz}, \mathrm{H}-16), 4.53$ (s, H-12), 2.29 (s, $\mathrm{H}_{3}-14$ ), 2.10 (m, H-17a), 1.94 (m, H-17b), 1.50 (m, $\left.\mathrm{H}_{2}-18\right), 1.44$ ( $\left.\mathrm{s}, \mathrm{H}_{3}-15\right), 1.38-1.25$ ( $\mathrm{m}, \mathrm{H}_{2}-19$ to $\left.\mathrm{H}_{2}-22\right), 0.87$ $\left(\mathrm{t}, J=7.0 \mathrm{~Hz}, \mathrm{H}_{3}-23\right) ;{ }^{13} \mathrm{C} \mathrm{NMR}\left(\mathrm{CDCl}_{3}, 125 \mathrm{MHz}\right): \delta 168.9$ (C-9), 162.62, 162.59, 162.56 (C-3, C-7, C-24), 151.1 (C-1), 138.7 (C-5), 119.9 (C-13), 113.2 (C-8), 108.0 (C-4), 94.3 (C-6), 80.8 (C-11), 79.1 (C-16), 69.9 (C-12), 33.3 (C-17), 31.8 (C-21), 29.3 (C-19), 29.1 (C-20), 24.1 (C-18), 22.6 (C-22), 22.6 (C-15), 19.9 (C-14), 14.1 (C-23), HRFABMS: $m / z 387.1800[\mathrm{M}+\mathrm{H}]^{+}\left(\mathrm{C}_{22} \mathrm{H}_{27} \mathrm{O}_{6}\right.$ requires 387.1808).

Pitholide B (2): luminous yellow oil; $[\alpha]_{\mathrm{D}}^{25}-741(c$, $0.23, \mathrm{MeOH}) ;{ }^{1} \mathrm{H} \mathrm{NMR}\left(\mathrm{CDCl}_{3}, 500 \mathrm{MHz}\right) \delta 9.71(s$, H-24), 7.99 ( $s, \mathrm{H}-4), 7.76(s, \mathrm{H}-1), 4.46(s, \mathrm{H}-12), 2.96(t$, $J=7.5 \mathrm{~Hz}, \mathrm{H}-17), 2.36\left(\mathrm{H}_{3}-14\right), 1.64\left(m, \mathrm{H}_{2}-18\right), 1.40(s$, $\left.\mathrm{H}_{3}-15\right), 1.34-1.20\left(m, \mathrm{H}_{2}-19\right.$ to $\left.\mathrm{H}_{2}-22\right), 0.87(t, J=7.0 \mathrm{~Hz}$, $\left.\mathrm{H}_{3}-23\right) ;{ }^{13} \mathrm{C}$ NMR $\left(\mathrm{CDCl}_{3}, 125 \mathrm{MHz}\right) \delta 198.6(\mathrm{C}-16)$, 186.8 (C-24), 170.7 (C-9), 166.9 (C-7), 164.2 (C-3), 152.8 (C-1), 143.9 (C-5), 119.5 (C-13), $117.6(\mathrm{C}-8), 107.9$ (C-4), 107.4 (C-6), 83.0 (C-11), 68.9 (C-12), 42.4 (C-17), 31.7 (C-21), 29.1 (C-19), 29.1 (C-20), 23.6 (C-18), 22.6 (C-22), 22.4 (C-15), 20.2 (C-14), $14.1(\mathrm{C}-23),{ }^{1} \mathrm{H}$ NMR $\left(\mathrm{CD}_{3} \mathrm{OD}\right.$, $500 \mathrm{MHz}) \delta 9.78(s, \mathrm{H}-24), 8.02(s, \mathrm{H}-4), 7.89(d, J=2.0 \mathrm{~Hz}$, $\mathrm{H}-1), 4.52$ ( $s, \mathrm{H}-12), 2.85$ ( $\left.m, \mathrm{H}_{2}-17\right), 2.37$ ( $\left.s, \mathrm{H}-14\right), 1.62$ ( $\left.m, \mathrm{H}_{2}-18\right), 1.37\left(s, \mathrm{H}_{3}-15\right), 1.37-1.29\left(m, \mathrm{H}_{2}-19\right.$ to $\left.\mathrm{H}_{2}-22\right)$, $0.89\left(t, J=7.0 \mathrm{~Hz}, \mathrm{H}_{3}-23\right) ;{ }^{13} \mathrm{C}$ NMR $\left(\mathrm{CD}_{3} \mathrm{OD}, 125 \mathrm{MHz}\right) \delta$ 200.7 (C-17), 187.8 (C-24), 172.9 (C-9), 166.6 (C-7), 166.4 (C-3), 154.9 (C-1), 147.2 (C-5), 122.0 (C-13), 119.6 (C-8), 108.4 (C-6), 107.8 (C-4), 84.9 (C-11), 69.7 (C-12), 43.5 (C-17), 30.3 (C-19), 32.9 (C-21), 30.3 (C-20), 24.9 (C-18), 23.7 (C-22), 22.5 (C-17), 20.0 (C-14), 14.4 (C-23); FABMS $\mathrm{m} / \mathrm{z}: 387[\mathrm{M}+\mathrm{H}]^{+}$.

Pitholide D (3): luminous yellow oil; $[\alpha]_{\mathrm{D}}{ }^{25}-980(c$, $0.077, \mathrm{MeOH}) ;{ }^{1} \mathrm{H}$ NMR $\left(\mathrm{CDCl}_{3}, 300 \mathrm{MHz}\right) \delta 7.30(s$, $\mathrm{H}-1), 6.72(s, \mathrm{H}-4), 6.10(s, \mathrm{H}-6), 4.41(s, \mathrm{H}-12), 2.94(m$, $\left.\mathrm{H}_{2}-17\right), 2.18\left(s, \mathrm{H}_{3}-14\right), 1.44\left(s, \mathrm{H}_{3}-15\right), 1.60(2 \mathrm{H}, m, \mathrm{H}-19)$, $1.39-1.22\left(m, \mathrm{H}_{2}-19\right.$ to $\left.\mathrm{H}_{2}-22\right), 0.88\left(t, J=6.7 \mathrm{~Hz}, \mathrm{H}_{3}-23\right)$; ${ }^{13} \mathrm{C} \mathrm{NMR}\left(\mathrm{CDCl}_{3}, 75 \mathrm{MHz}\right) \delta 197.6(\mathrm{C}-16), 175.2(\mathrm{C}-7)$, 161.1 (C-3), 149.0 (C-1), 145.0 (C-5), 119.0 (C-13), 111.9 (C-8), 109.7 (C-4), 102.2 (C-6), 82.5 (C-11), 69.0 (C-12), 41.5 (C-17), 31.7 (C-21), 29.2 (C-19), 29.2 (C-20), 24.3 (C-15), 23.7 (C-18), 22.6 (C-22), 19.6 (C-14), 14.1 (C-23), C-9 signal was ambiguous due to weak signal intensity; FABMS $m / z: 359[\mathrm{M}+\mathrm{H}]^{+}$.

Pestalotin (LL-P880 $\alpha)(4)$ : white solid; $[\alpha]_{D}{ }^{25}-82.3$ $(c, 0.78, \mathrm{MeOH}) ;{ }^{1} \mathrm{H} \mathrm{NMR}\left(\mathrm{CDCl}_{3}, 300 \mathrm{MHz}\right): \delta 5.15(d$, $J=1.8 \mathrm{~Hz}, \mathrm{H}-3), 4.30(d d d, J=12.6,3.9,3.9 \mathrm{~Hz}, \mathrm{H}-6)$, $3.76\left(s, 4-\mathrm{OCH}_{3}\right), 3.64\left(m, \mathrm{H}-1^{\prime}\right), 2.80(d d d, J=16.8,12.6$, $1.8 \mathrm{~Hz}, \mathrm{H}-5 \mathrm{a}), 2.25$ ( $d d, J=16.8,3.9 \mathrm{~Hz}, \mathrm{H}-5 \mathrm{~b}$ ), 1.68-1.32 $\left(m, \mathrm{H}_{2}-2^{\prime}, \mathrm{H}_{2}-3^{\prime}, \mathrm{H}_{2}-4^{\prime}\right), 0.92\left(t, J=7.2 \mathrm{~Hz}, \mathrm{H}_{3}-5^{\prime}\right) ;{ }^{13} \mathrm{C}$ NMR (CDCl $3,75 \mathrm{MHz}): \delta 173.1(\mathrm{C}-4), 166.7$ (C-2), 90.0 (C-3), $78.4(\mathrm{C}-6), 72.5\left(\mathrm{C}-1^{\prime}\right), 56.2\left(4-\mathrm{OCH}_{3}\right), 32.4\left(\mathrm{C}-2^{\prime}\right)$, 
$29.6(\mathrm{C}-5), 27.6\left(\mathrm{C}-3^{\prime}\right), 22.6\left(\mathrm{C}-4^{\prime}\right), 14.0\left(\mathrm{C}-5^{\prime}\right)$; FABMS $m / z: 215[\mathrm{M}+\mathrm{H}]^{+}$.

PC-2 (5): white solid; $[\alpha]_{\mathrm{D}}{ }^{25}+76.5(c, 0.62, \mathrm{MeOH}) ;{ }^{1} \mathrm{H}$ NMR ( $\left.\mathrm{CD}_{3} \mathrm{OD}, 300 \mathrm{MHz}\right) \delta 6.19(d, J=2.3 \mathrm{~Hz}, \mathrm{H}-5), 5.55$ $(d, J=2.3 \mathrm{~Hz}, \mathrm{H}-3), 4.32$ ( $\left.d d, J=7.8,4.8 \mathrm{~Hz}, \mathrm{H}-1^{\prime}\right), 3.86$ $\left(s, 4-\mathrm{OCH}_{3}\right), 1.78\left(m, \mathrm{H}-2^{\prime} \mathrm{a}\right), 1.65\left(m, \mathrm{H}-2^{\prime} \mathrm{b}\right), 1.44-1.28$ $\left(m, \mathrm{H}_{2}-3^{\prime}, \mathrm{H}_{2}-4^{\prime}\right), 0.92\left(t, J=7.2 \mathrm{~Hz}, \mathrm{H}_{3}-5^{\prime}\right) ;{ }^{13} \mathrm{C} \mathrm{NMR}$ $\left(\mathrm{CD}_{3} \mathrm{OD}, 75 \mathrm{MHz}\right): \delta 173.8(\mathrm{C}-4), 169.0(\mathrm{C}-6), 167.1$ (C-2), 99.8 (C-5), $88.6(\mathrm{C}-3), 71.1\left(\mathrm{C}-1^{\prime}\right), 57.0\left(4-\mathrm{OCH}_{3}\right)$, 35.8 (C-2'), $28.4\left(\mathrm{C}-3^{\prime}\right), 23.5$ (C-4'), 14.3 (C-5'); FABMS $m / z: 213[\mathrm{M}+\mathrm{H}]^{+}$.

LL-P880 $\beta$ (6): white solid; $[\alpha]_{\mathrm{D}}{ }^{25}-61.2(c, 0.23$, $\mathrm{MeOH}) ;{ }^{1} \mathrm{H} \mathrm{NMR}\left(\mathrm{CDCl}_{3}, 500 \mathrm{MHz}\right) \delta 5.14(d, J=1.7 \mathrm{~Hz}$, $\mathrm{H}-3), 4.51$ ( $d d d, J=12.8,3.9,3.9 \mathrm{~Hz}, \mathrm{H}-6), 3.80$ ( $\left.m, \mathrm{H}-2^{\prime}\right)$, $3.76\left(s, 4-\mathrm{OCH}_{3}\right), 3.49\left(m, \mathrm{H}-1^{\prime}\right), 2.89(m, \mathrm{H}-5 \mathrm{a}), 2.32(d d$, $J=17.2,3.9 \mathrm{~Hz}, \mathrm{H}-5 \mathrm{~b}), 1.63$ (m, H-3'a), 1.52 (m, H-3'b), $1.52\left(m, \mathrm{H}-4^{\prime} \mathrm{a}\right), 1.40\left(m, \mathrm{H}-4^{\prime} \mathrm{b}\right), 0.95(t, J=7.2 \mathrm{~Hz}$, $\left.5^{\prime}-\mathrm{H}_{3}\right) ;{ }^{13} \mathrm{C} \mathrm{NMR}\left(\mathrm{CDCl}_{3}, 75 \mathrm{MHz}\right) \delta 173.3(\mathrm{C}-4), 166.3$ (C-2), 89.8 (C-3), 78.0 (C-6), 73.8 (C-1'), 71.0 (C-2'), 56.2 $\left(4-\mathrm{OCH}_{3}\right), 36.0\left(\mathrm{C}-3^{\prime}\right), 29.4(\mathrm{C}-5), 18.8\left(\mathrm{C}-4^{\prime}\right), 13.9\left(\mathrm{C}^{\prime} 5^{\prime}\right)$; FABMS $m / z: 231[\mathrm{M}+\mathrm{H}]^{+}$.

Tyrosol (7): white solid; ${ }^{1} \mathrm{H}$ NMR $\left(\mathrm{CDCl}_{3}, 300 \mathrm{MHz}\right)$ $\delta 7.10(d, J=8.4 \mathrm{~Hz}, \mathrm{H}-3, \mathrm{H}-5), 6.79(d, J=8.4 \mathrm{~Hz}, \mathrm{H}-2$, $\mathrm{H}-6), 3.83\left(t, J=6.6 \mathrm{~Hz}, \mathrm{H}_{2}-2^{\prime}\right), 2.81\left(t, J=6.6 \mathrm{~Hz}, \mathrm{H}_{2}-1^{\prime}\right)$; ${ }^{13} \mathrm{C}$ NMR $\left(\mathrm{CDCl}_{3}, 75 \mathrm{MHz}\right) \delta 154.3(\mathrm{C}-4), 115.4(\mathrm{C}-3$, C-5), 130.1 (C-2, C-6), 130.3 (C-1'), 63.8 (C-2'), 38.2 $\left(\mathrm{C}-1^{\prime}\right)$; FABMS $m / z: 139[\mathrm{M}+\mathrm{H}]^{+}$.

4-Oxo-4H-pyran-3-acetic acid (8): white solid; ${ }^{1} \mathrm{H}$ NMR $\left(\mathrm{CD}_{3} \mathrm{OD}, 500 \mathrm{MHz}\right) \delta 8.11(d, J=1.0 \mathrm{~Hz}, \mathrm{H}-2), 8.04(d d$, $J=7.0,1.0 \mathrm{~Hz}, \mathrm{H}-6), 6.39(d, J=7.0 \mathrm{~Hz}, \mathrm{H}-5), 3.52(s$, $\left.\mathrm{H}_{2}-7\right) ;{ }^{13} \mathrm{C} \mathrm{NMR}\left(\mathrm{CD}_{3} \mathrm{OD}, 125 \mathrm{MHz}\right.$, $\delta 180.2(\mathrm{C}-4), 174.0$ (C-8, signal was not clearly observed but assigned on the basis of the HMBC correlation), 158.6 (C-2), 156.9 (C-6), 125.8 (C-3), 117.1 (C-5), 31.6 (C-7); ${ }^{1} \mathrm{HNMR}\left(\mathrm{CDCl}_{3}\right.$, $300 \mathrm{MHz}) \delta 7.92(s, \mathrm{H}-2), 7.87(d, J=5.4 \mathrm{~Hz}, \mathrm{H}-6), 6.51$ $(d, J=5.4 \mathrm{~Hz}, \mathrm{H}-5), 3.47\left(s, \mathrm{H}_{2}-7\right) ;{ }^{13} \mathrm{CNMR}\left(\mathrm{CDCl}_{3}\right.$, $75 \mathrm{MHz}$,) $\delta 179.5$ (C-4), 156.4 (C-2), 154.5 (C-6), 123.8 (C-3), 116.7 (C-5), 29.7 (C-7); ${ }^{1} \mathrm{H}$ NMR $\left(\mathrm{D}_{2} \mathrm{O}, 300 \mathrm{MHz}\right.$, referenced to internal dioxane at $\delta 3.53) \delta 7.96(s, \mathrm{H}-2)$, $7.93(d, J=5.7 \mathrm{~Hz}, \mathrm{H}-6), 6.32(d, J=5.7 \mathrm{~Hz}, \mathrm{H}-5), 3.23$ $\left(s, \mathrm{H}_{2}-7\right) ;{ }^{13} \mathrm{CNMR}\left(\mathrm{D}_{2} \mathrm{O}, 75 \mathrm{MHz}\right.$, referenced to internal dioxane at $\delta 67.4) \delta 181.8(\mathrm{C}-4), 176.2(\mathrm{C}-8), 159.4(\mathrm{C}-2)$, 157.7 (C-6), 124.5 (C-3), 116.5 (C-5), 32.1 (C-7); FABMS $m / z: 155[\mathrm{M}+\mathrm{H}]^{+}$.

Acknowledgements Financial support from the National Research Council (Research Grant NRC 12-032) is gratefully acknowledged.

\section{Compliance with Ethical Standards}

Conflict of interest The authors declare no conflict of interest.

Open Access This article is distributed under the terms of the Creative Commons Attribution 4.0 International License (http://creativeco mmons.org/licenses/by/4.0/), which permits unrestricted use, distribution, and reproduction in any medium, provided you give appropriate credit to the original author(s) and the source, provide a link to the Creative Commons license, and indicate if changes were made.

\section{References}

1. Y. Zhang, J. Mu, Y. Feng, Y. Kang, J. Zhang, P.J. Gu, Y. Wang, L.F. Ma, Y.H. Zhu, Mar. Drugs 7, 97-112 (2009)

2. J. Zhao, L. Zhou, J. Wang, T. Shan, L. Zhong, X. Liu, X. Gao, in Current research, technology and education topics in applied microbiology and microbial biotechnology, ed. by A. MëndezVilas, (Badajoz, Formatex Research Center, 2010), pp. 567-576

3. K.G.E. Padmathilake, H.M.S.K.H. Bandara, M.M. Qader, N.S. Kumar, L. Jayasinghe, H. Masubuti, Y. Fujimoto, Nat. Prod. Commun. 12, 489-490 (2017).

4. K.G.N.P. Piyasena, W.A.R.T. Wickramarachchi, N.S. Kumar, L. Jayasinghe, Y. Fujimoto, Mycology 6, 158-160 (2015)

5. M.M. Qader, N.S. Kumar, L. Jayasinghe, Y. Fujimoto, Med. Aromat. Plants 5, 225-231 (2016)

6. M.M. Qader, N.S. Kumar, L. Jayasinghe, H. Araya, Y. Fujimoto, Mycology 8, 17-20 (2017)

7. M.V.K. Munasinghe, N.S. Kumar, L. Jayasinghe, Y. Fujimoto, J. Biol. Act. Prod. Nat. 7, 475-479 (2017)

8. G.R.N. Rathnayake, N.S. Kumar, L. Jayasinghe, H. Araya, Y. Fujimoto, Nat. Prod. Res. 32, 2483-2486 (2018)

9. D. Thanabalasingam, N.S. Kumar, L. Jayasinghe, Y. Fujimoto, Nat. Prod. Commun. 10, 1659-1660 (2015)

10. T. Sritharan, N.S. Kumar, L. Jayasinghe, H. Araya, Y. Fujimoto, Nat. Prod. Commun. (2019). https://doi.org/10.1177/1934578X19 851969

11. S.M. Barbalho, P.C. Bueno, D.S. Delazari, E.L. Guiguer, D.P. Coqueiro, A.C. Araújo, M.D. de Souza, F.M. Farinazzi-Machado, C.G. Mendes, M. Groppo, J. Med. Food 18, 385-391 (2015)

12. P. Milind, M. Preethi, Int. J. Res. Ayurveda Pharm. 6, 544-550 (2015)

13. A.L. Homans, A. Fuchs, J. Chromatogr. 51, 327-329 (1970)

14. B. Tepe, O. Eminagaoglu, H.A. Akpulat, E. Aydin, Food Chem. 100, 985-989 (2007)

15. A.V. Krishnaraju, T.V.N. Rao, D. Sundararaju, M. Vanisree, H.S. Tsay, G.V. Subbaraju, Int. J. Appl. Sci. Eng. 3, 125-134 (2005)

16. T.D.G. Baratelli, A.C.C. Gomes, L.A. Wessjohann, R.M. Kuster, N.K. Simas, Biochem. Syst. Ecol. 41, 119-125 (2012)

17. B. Nickavar, L. Abolhasani, H. Izadpanah, Iran. J. Pharm. Res. 7, 297-303 (2008)

18. J.M. Andrews, J. Antimicrob. Chemother. 48(Suppl 1), 5-16 (2001)

19. G.Y.S. Wang, B.M. Borgeson, P. Crews, Tetrahedron Lett. 38, 8449-8452 (1997)

20. Y. Kimura, A. Suzuki, S. Tamura, Agric. Biol. Chem. 44, 451-452 (1980)

21. J. Zhang, D.P. Curran, J. Chem. Soc. Perkin Trans. 1, 2627-2631 (1991)

22. Y. Kimura, W.J. McGahren, A. Suzuki, S. Tamura, Agric. Biol. Chem. 42, 1625-1626 (1978)

23. L. Rahbaek, S. Sperry, J.C. Frisvad, T.O. Larsen, Biochem. Syst. Ecol. 31, 313-317 (2003)

24. W.J. McGahren, G.A. Ellestad, G.O. Morton, M.P. Kunstmann, P. Mullen, J. Org. Chem. 38, 3542-3544 (1973)

25. V.S. Mallula, B. Srinivas, P.R. Krishna, Tetrahedron Lett. 56, 1115-1117 (2015)

26. J. Rovirosa, A. Diaz-Marrero, J. Darias, K. Painemal, A.S. Martin, J. Chil. Chem. Soc. 51, 775-778 (2006) 
27. M.J. Salvatore, O.D. Hensens, D.L. Zink, J. Liesch, C. Dufresne, J.G. Ondeyka, T.M. Jürgens, R.P. Borris, S. Raghoobar, E. McCauley, L. Kong, J. Nat. Prod. 57, 755-760 (1994)

28. A. Andolfi, L. Maddau, B.T. Linaldeddu, B. Scanu, A. Cimmino, S. Basso, A. Evidente, Phytochem. Lett. 10, 40-45 (2014)

29. Y.W. Hsu, L.C. Hsu, Y.H. Liang, Y.H. Kuo, T.M. Pan, J. Agric. Food Chem. 59, 4512-4518 (2011)

30. S. Kanokmedhakul, K. Kanokmedhakul, P. Nasomjai, S. Louangsysouphanh, K. Soytong, M. Isobe, P. Kongsaeree, S. Prabpai, A. Suksamrarn, J. Nat. Prod. 69, 891-895 (2006)

31. G. Strobel, X. Yang, J. Sears, R. Kramer, R.S. Sidhu, W.M. Hess, Microbiology 142, 435-440 (1996)

32. J.R. Russell, J. Huang, P. Anand, K. Kucera, A.G. Sandoval, K.W. Dantzler, D. Hickman, J. Jee, F.M. Kimovec, D. Koppstein, D.H. Marks, Appl. Environ. Microbiol. 77, 6076-6084 (2011)

33. Y. Kimura, K. Katagiri, T. Inoue, S. Tamura, Agric. Biol. Chem. 35, 1313-1314 (1971)

34. D.Q. Luo, L. Zhang, B.Z. Shi, X.M. Song, Molecules 17, 8554$8560(2012)$

35. J.F. Tian, R.J. Yu, X.X. Li, H. Gao, L.D. Guo, J.S. Tang, X.S. Yao, Magn. Reson. Chem. 53, 866-871 (2015)
36. H.E. Ortega, Y.Y. Shen, K. TenDyke, N. Ríos, L. Cubilla-Ríos, Tetrahedron Lett. 55, 2642-2645 (2014)

37. G. Ding, Y. Qi, S. Liu, L. Guo, X. Chen, J. Antibiot. 65, 271-273 (2012)

38. W.A. Ayer, L.S. Trifonov, L.J. Hutchison, P. Chakravarty, Nat. Prod. Lett. 14, 405-410 (2000)

39. X. Li, Z. Guo, Z. Deng, J. Yang, K. Zou, Rec. Nat. Prod. 9, 503$508(2015)$

40. D. Rönsberg, A. Debbab, A. Mándi, V. Wray, H. Dai, T. Kurtán, P. Proksch, A.H. Aly, Tetrahedron Lett. 54, 3256-3259 (2013)

41. S. Akay, G. Ekiz, F. Kocabas, E.E. Hames-Kocabas, K.S. Korkma, E. Bedir, Phytochem. Lett. 7, 93-96 (2014)

42. J.Y. Xian, X.P. Lin, J.F. Wang, W. Al, C. Qin, Z.L. Zhong, Y.H. Liu, Nat. Prod. Res. Dev. 26, 1412-1415 (2014)

43. Z.Z. Li, X.L. Yang, Junwu Xuebao 33, 97-102 (2014)

44. H. Chen, M. Fujita, Q. Feng, J. Clardy, G.R. Fink, Proc. Natl. Acad. Sci. USA 101, 5048-5052 (2004)

45. Y.L. Zhang, H.M. Ge, F. Li, Y.C. Song, R.X Tan, Chem. Biodivers. 5, 2402-2407 (2008).

46. H.C. Dube, Mycopathol. Mycol. Appl. 44, 183-191 (1971)

\section{Affiliations}

\section{G. R. Nalin Rathnayake ${ }^{1} \cdot$ N. Savitri Kumar ${ }^{1} \cdot$ Lalith Jayasinghe $^{1} \cdot$ Hiroshi Araya $^{2} \cdot$ Yoshinori Fujimoto $^{1,2}$ \\ 1 National Institute of Fundamental Studies, Hantana Road, Kandy, Sri Lanka \\ 2 School of Agriculture, Meiji University, Kawasaki, Kanagawa 214-8571, Japan}

